\title{
RB1 Gene Deletion
}

National Cancer Institute

\section{Source}

National Cancer Institute. RB1 Gene Deletion. NCI Thesaurus. Code C146654.

A molecular abnormality referring to the loss of at least one copy of the RB1 gene. 